\title{
Efficient Lymph Node-Targeted Delivery of Personalized Cancer Vaccines with Reactive Oxygen Species-Inducing Reduced Graphene Oxide Nanosheets.
}

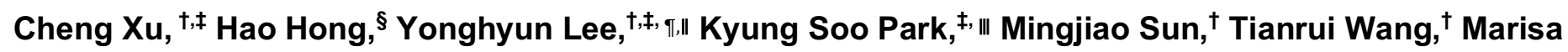
E. Aikins, ${ }^{\dagger, \ddagger}$ Yao Xu, ${ }^{\dagger, \ddagger}$ and James J. Moon ${ }^{\dagger, \ddagger}, \|, * *$

${ }^{\dagger}$ Department of Pharmaceutical Sciences, University of Michigan, Ann Arbor, MI 48109, United States.

${ }^{\ddagger}$ Biointerfaces Institute, University of Michigan, Ann Arbor, MI 48109, United States.

$\S$ Medical School of Nanjing University, 22 Hankou Road, Nanjing, Jiangsu 210093, China.

ॠ Department of Pharmacy, College of Pharmacy, Ewha Womans University, Seoul, 03760, South Korea.

"Pharmacy of Graduate School, Ewha Womans University, Seoul, 03760, South Korea.

IIDepartment of Biomedical Engineering, University of Michigan, Ann Arbor, MI 48109, United States.

*. Correspondence should be addressed to J.J.M. (moonjj@umich.edu). 

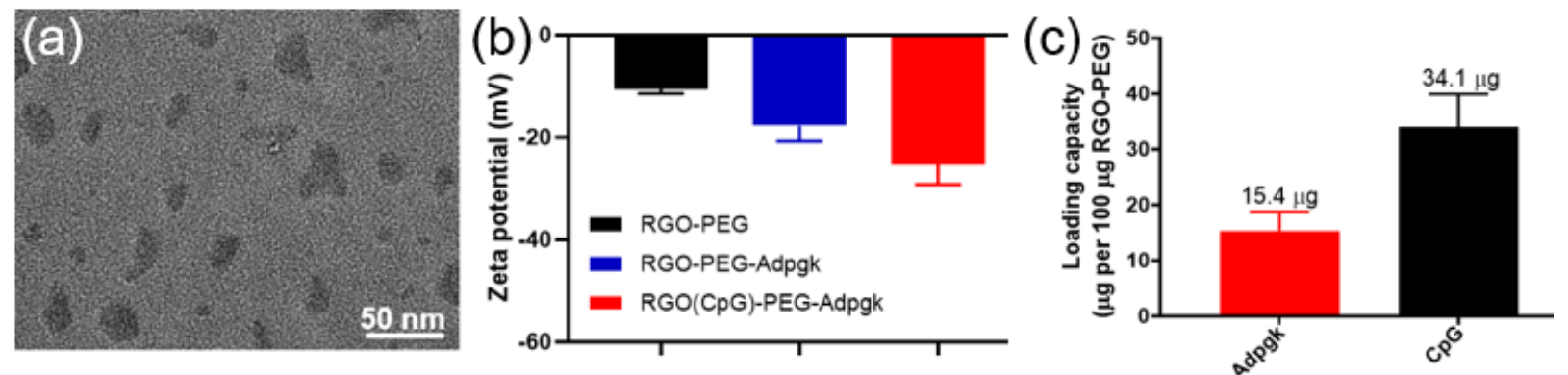

(d)
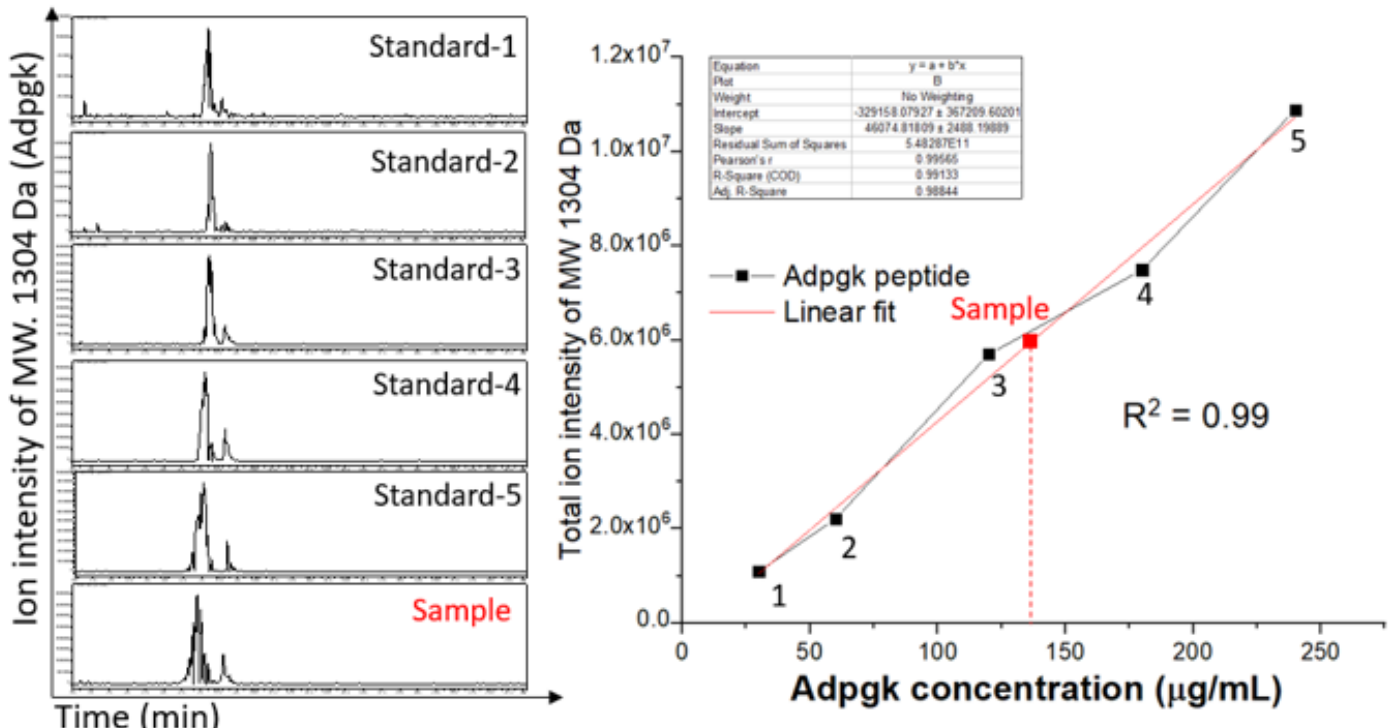

(e)
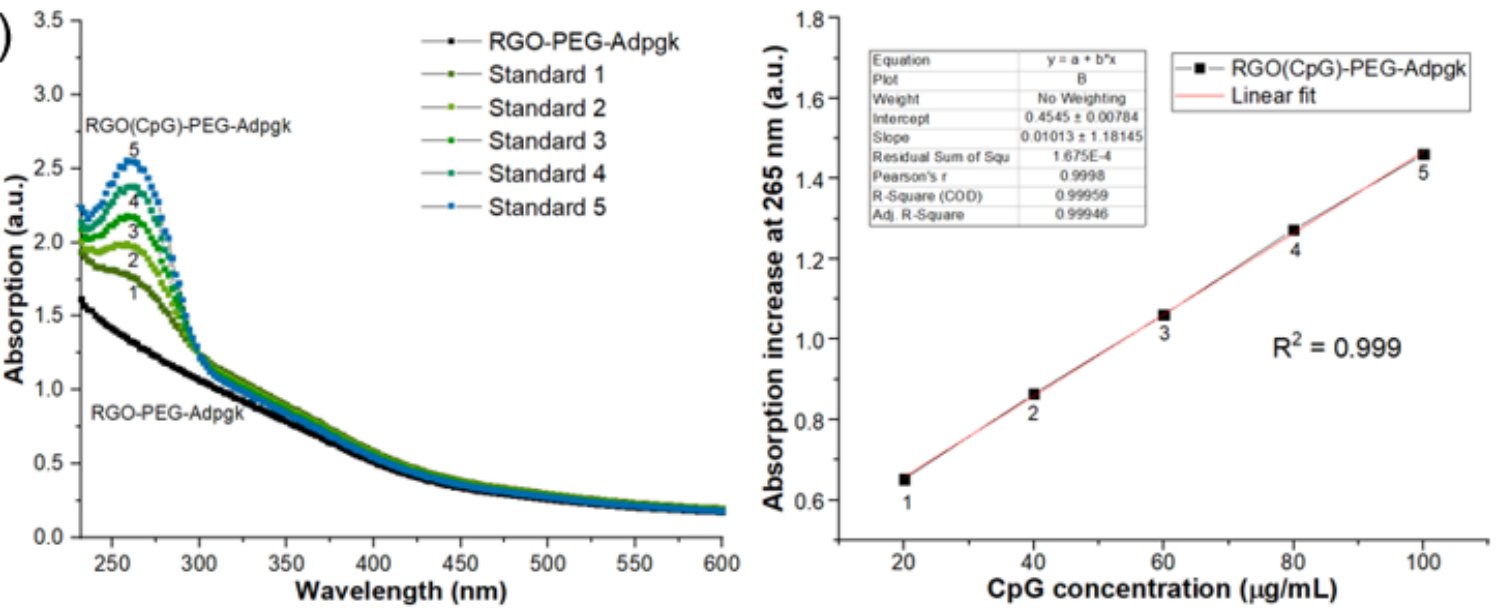

Figure S1. a, TEM of RGO-PEG. b, Surface zeta potential of RGO-PEG, RGO-PEG-Adpgk, and RGO(CpG)PEG-Adpgk. c, The amount of CpG and Adpgk neoantigen peptide loaded per $100 \mu \mathrm{g}$ of RGO-PEG in PBS at RT. d, mass spectrum and standard curve of Adpgk peptide (concentration vs. ion intensity of molecular weight of $1304 \mathrm{Da}$ ) for the quantification of Adpgk peptide. excess glutathione (100 mM) was added to cleavage and release Adpgk peptide from RGO(CpG)-PEG. e, UV-vis spectrum of RGO-PEG-Adpgk and RGO(CpG)-PEGAdpgk with various CpG concentrations. Inset: the standard curve for CpG quantification (concentrations vs. absorption increase at $265 \mathrm{~nm}$ between RGO-PEG-Adpgk and RGO(CpG)-PEG-Adpgk). 

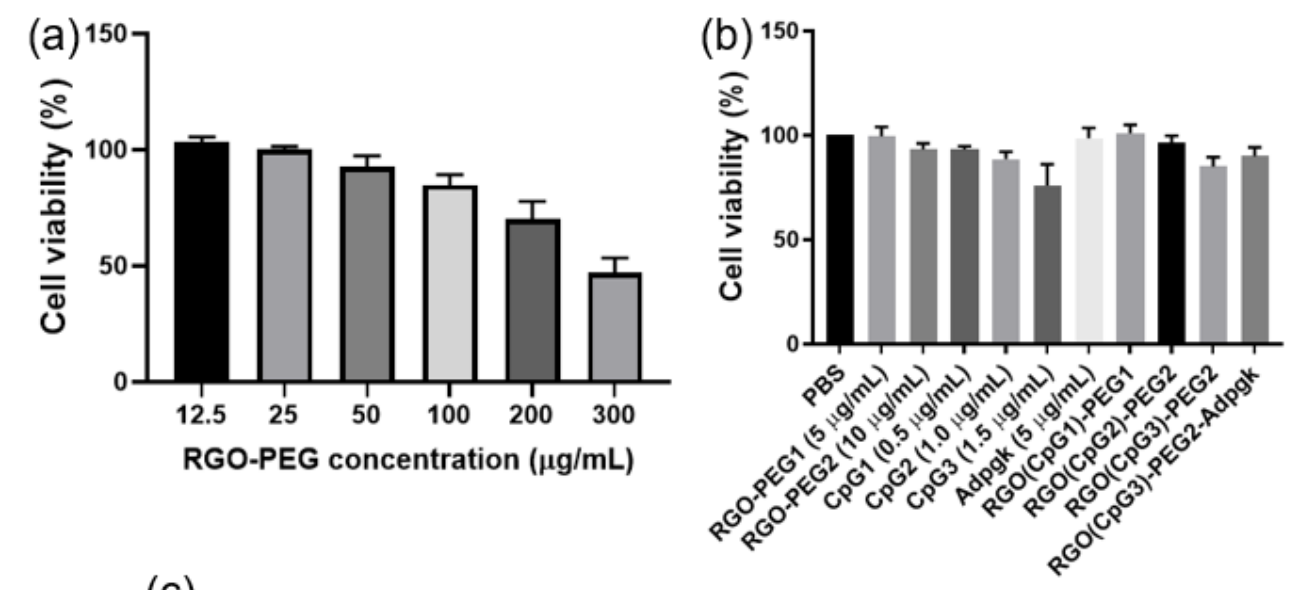

(c)

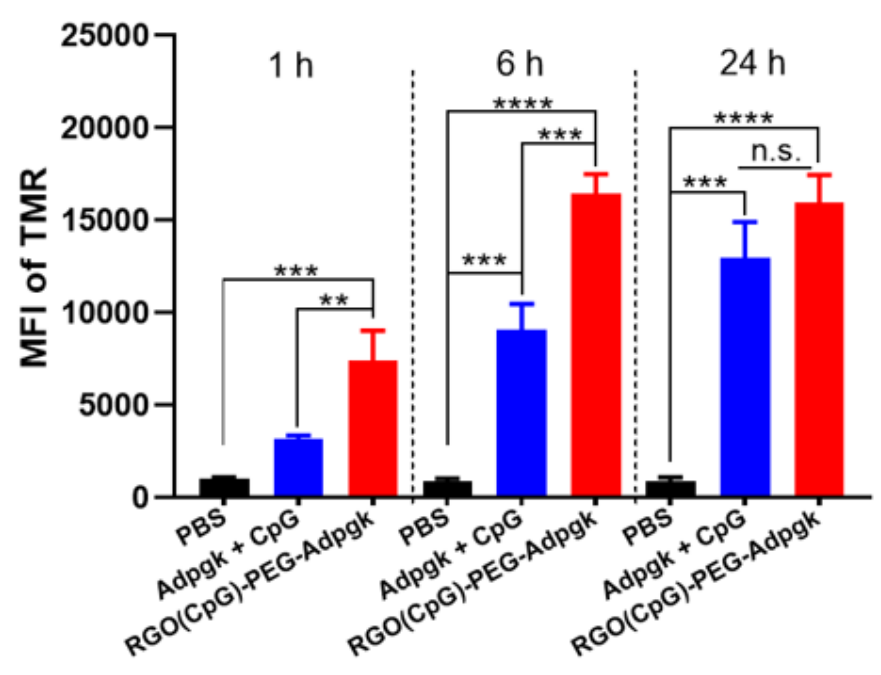

Figure S2. a, Viability of BMDCs after $24 \mathrm{~h}$ incubation with varying concentrations of RGO-PEG. b, Viability of BMDCs after $24 \mathrm{~h}$ incubation with varying concentrations of RGO-PEG, soluble CpG, soluble Adpgk, RGO(CpG)PEG, and RGO(CpG)-PEG-Adpgk. c, BMDC uptake of free Adpgk-tetramethylrhodamine (TMR) $(4 \mu \mathrm{g} / \mathrm{mL}$ ) with CpG $(0.5 \mu \mathrm{g} / \mathrm{mL})$ or RGO(CpG)-PEG-Adpgk-TMR $(4 \mu \mathrm{g} / \mathrm{mL})$ after incubation with BMDCs for $1 \mathrm{~h}, 6 \mathrm{~h}$, or $24 \mathrm{~h}$. The concentrations of $\mathrm{CpG}$ and Adpgk in RGO(CpG)-PEG or RGO(CpG)-PEG-Adpgk were the same with the corresponding soluble $\mathrm{CpG}$ and Adpgk group. Data represent mean \pm SEM from a representative experiment ( $\mathrm{n}$ $=3$ ). Data was analyzed by one-way ANOVA with Tukey's HSD multiple comparison post hoc test, ${ }^{* *} P<0.01$, ${ }^{* * *} \mathrm{P}<0.001,{ }^{* * * *} \mathrm{P}<0.0001$. 
(a)
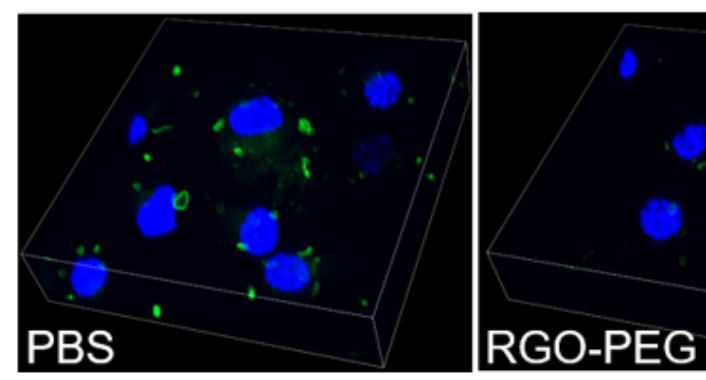

(b)

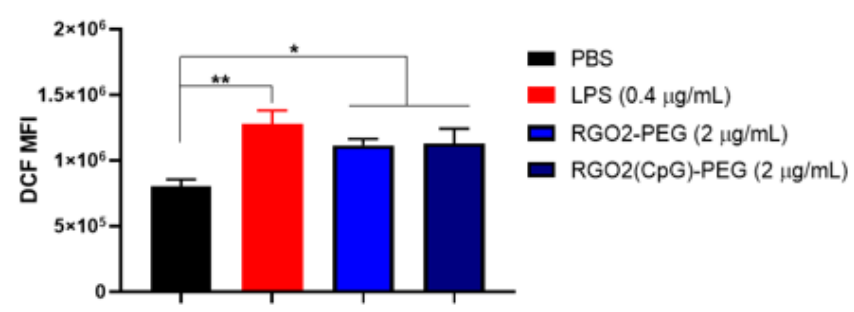

(c)

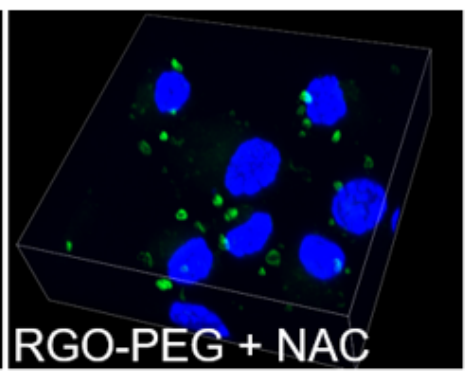

(d)
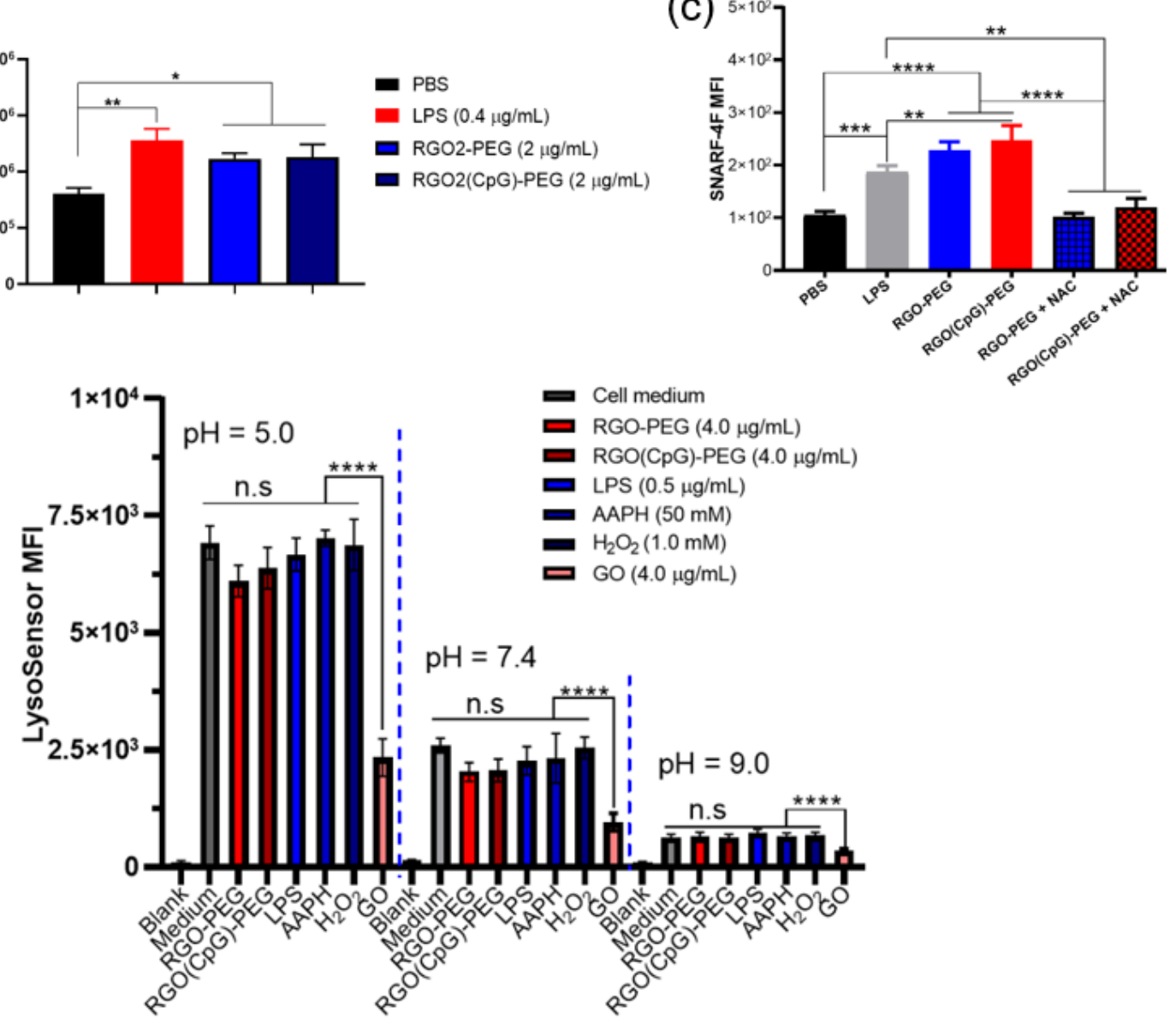

Figure S3. a, Confocal microscopy images of BMDCs incubated for $4 \mathrm{~h}$ with PBS, RGO-PEG $(4 \mu \mathrm{g} / \mathrm{mL})$, or RGO-PEG $(4 \mu \mathrm{g} / \mathrm{mL})+\mathrm{NAC}(5 \mathrm{mM})$, followed by staining with LysoSensor (green). b. Intracellular DCF fluorescence level in BMDMs after $24 \mathrm{~h}$ incubation with LPS, RGO-PEG, or RGO(CpG)-PEG. c, SNARF-4F-5(and 6-) carboxylic acid fluorescence intensity in BMDCs after incubation with PBS, LPS $(0.4 \mu \mathrm{g} / \mathrm{mL}$ ), RGO-PEG $(4 \mu \mathrm{g} / \mathrm{mL}), R G O(C p G)$-PEG (4 $\mu \mathrm{g} / \mathrm{mL})$, RGO-PEG + NAC (5 mM), RGO(CpG)-PEG + NAC (5 mM) for 4 h. d, Changes in LysoSensor fluorescence signal after incubation with RGO-PEG, RGO(CpG)-PEG, LPS, AAPH (2,2'Azobis(2-amidinopropane) dihydrochloride), $\mathrm{H}_{2} \mathrm{O}_{2}$, or $\mathrm{GO}$ in cell medium with different pH conditions. Data represent mean \pm SEM from a representative experiment $(n=4)$. Data was analyzed by one-way ANOVA with Tukey's HSD multiple comparison post hoc test, ${ }^{*} \mathrm{P}<0.05$. ${ }^{* *} \mathrm{P}<0.01$, ${ }^{* * *} \mathrm{P}<0.0001,{ }^{* * * *} \mathrm{P}<0.0001$. 

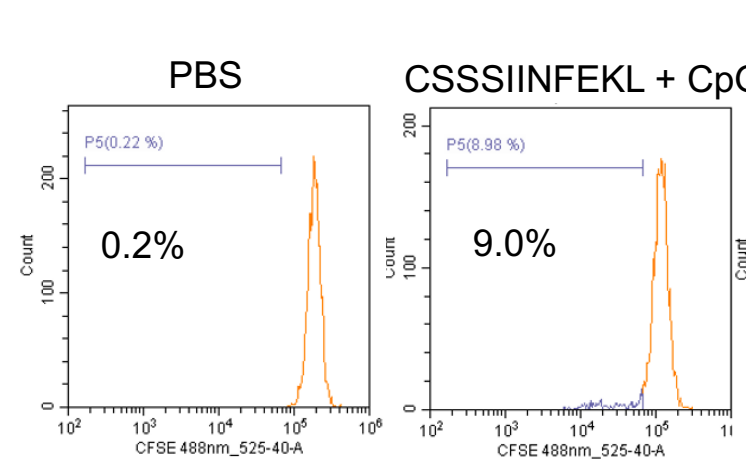

CSSSIINFEKL + CpG

RGO(CpG)-PEG-

$\mathrm{RGO}(\mathrm{CpG})$-PEGCSSSIINFEKL CSSSIINFEKL + NAC
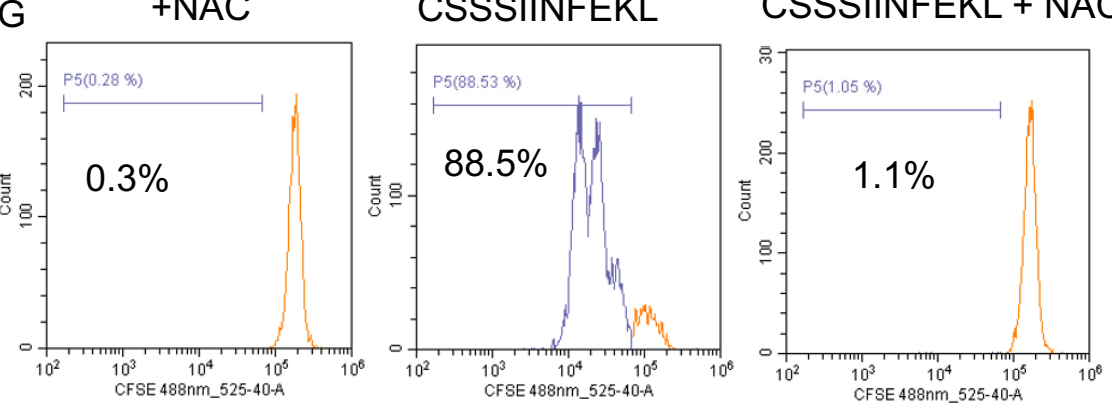

Figure S4. CFSE dilution assay. BMDCs were incubated with PBS, soluble CSSSIINFEKL + CpG, CSSSIINFEKL + CpG with NAC (5 mM), RGO(CpG)-PEG-CSSSIINFEKL, or RGO(CpG)-PEG-CSSSIINFEKL with NAC (5 mM). All groups had equivalent concentration of peptide at $5 \mu \mathrm{g} / \mathrm{mL}$ and $\mathrm{CpG}$ at $1 \mu \mathrm{g} / \mathrm{mL}$. After 24 $\mathrm{h}, \mathrm{BMDC}$ sere co-cultured with CFSE-labeled OT-1 T cells. After $48 \mathrm{~h}$, CFSE dilution was quantified by flow cytometric analysis.

(a)

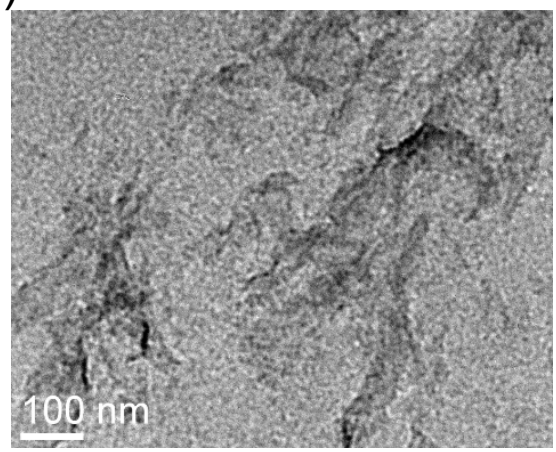

(b)

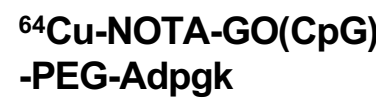

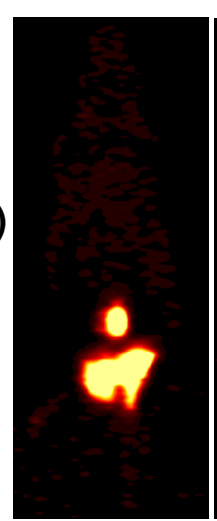

$0.5 \mathrm{~h}$

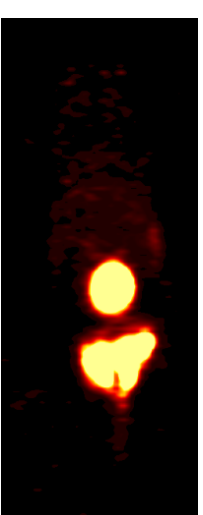

$3 \mathrm{~h}$

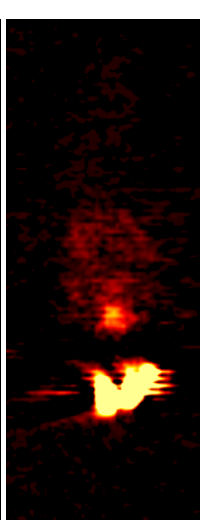

$24 \mathrm{~h}$
$15 \%$ ID/g

$0 \%$ ID/g

(c)

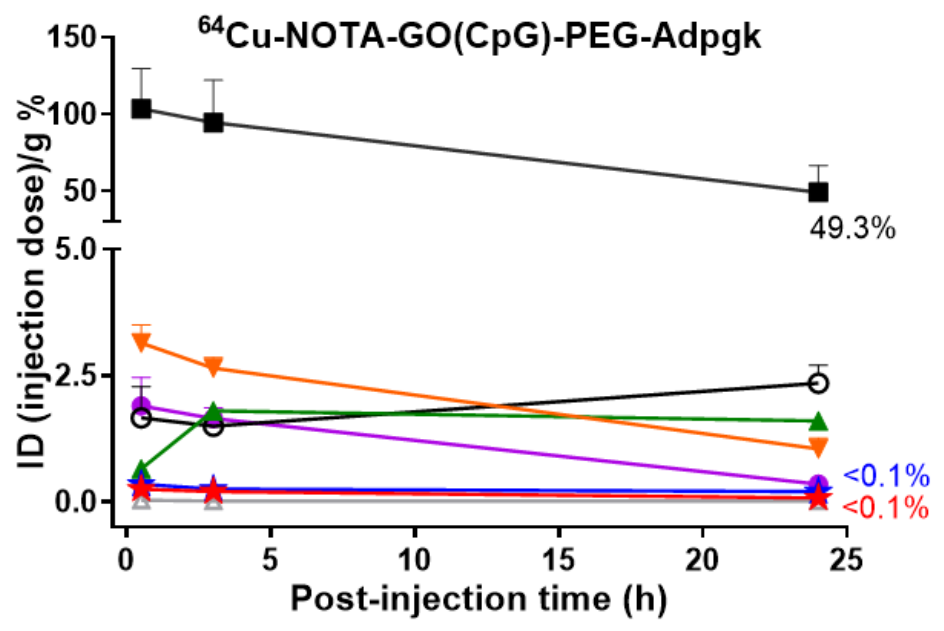

Figure S5. a, TEM image of GO-PEG, showing $~ 200-300 \mathrm{~nm}$ in size. b, Serial PET images of C57BL/6 mice administered SC with ${ }^{64} \mathrm{Cu}$-NOTA-GO(CpG)-PEG-Adpgk. c, Time-radioactivity curves of injection site, inguinal and axillary LNs, kidney, intestine, liver, blood, and muscle after SC administration of ${ }^{64} \mathrm{Cu}-\mathrm{NOTA}-\mathrm{GO}(\mathrm{CpG})-$ PEG-Adpgk. 

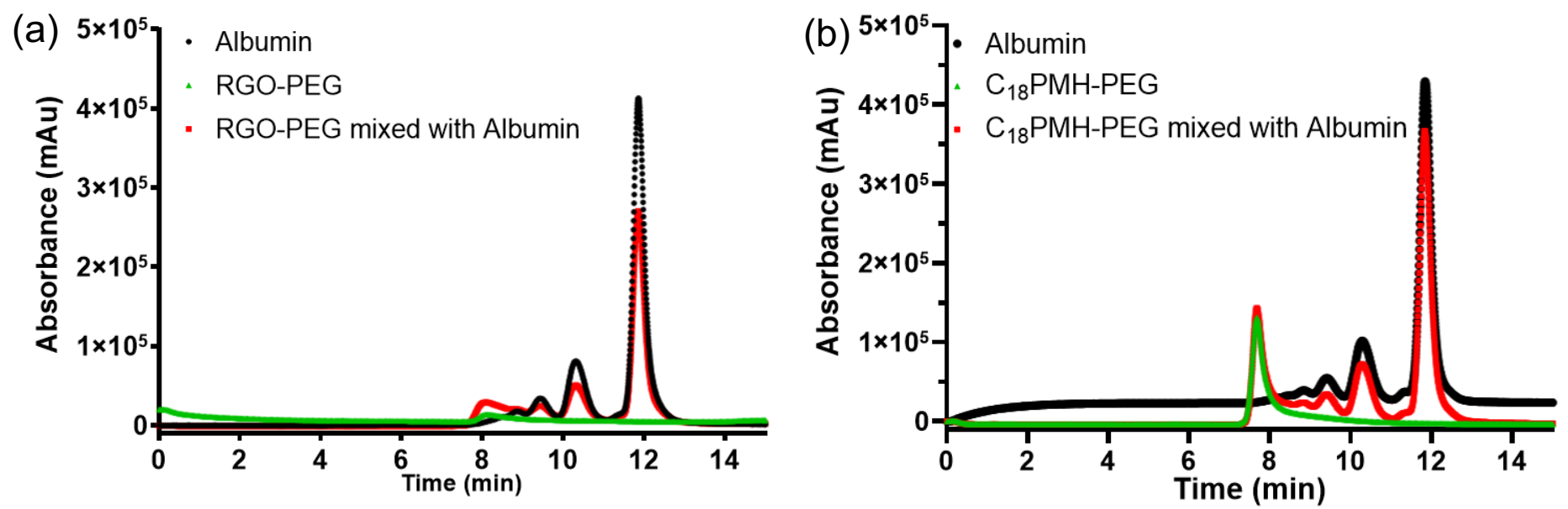

Figure S6. Gel permeation chromatography (GPC) of mouse albumin $(0.5 \mathrm{mg} / \mathrm{mL})$ with (a) RGO-PEG $(0.5$ $\mathrm{mg} / \mathrm{mL})$ or $(\mathbf{b}) \mathrm{C}_{18} \mathrm{PMH}-\mathrm{PEG}(0.5 \mathrm{mg} / \mathrm{mL})$ after incubation of $4 \mathrm{~h}$ at $37^{\circ} \mathrm{C}$.

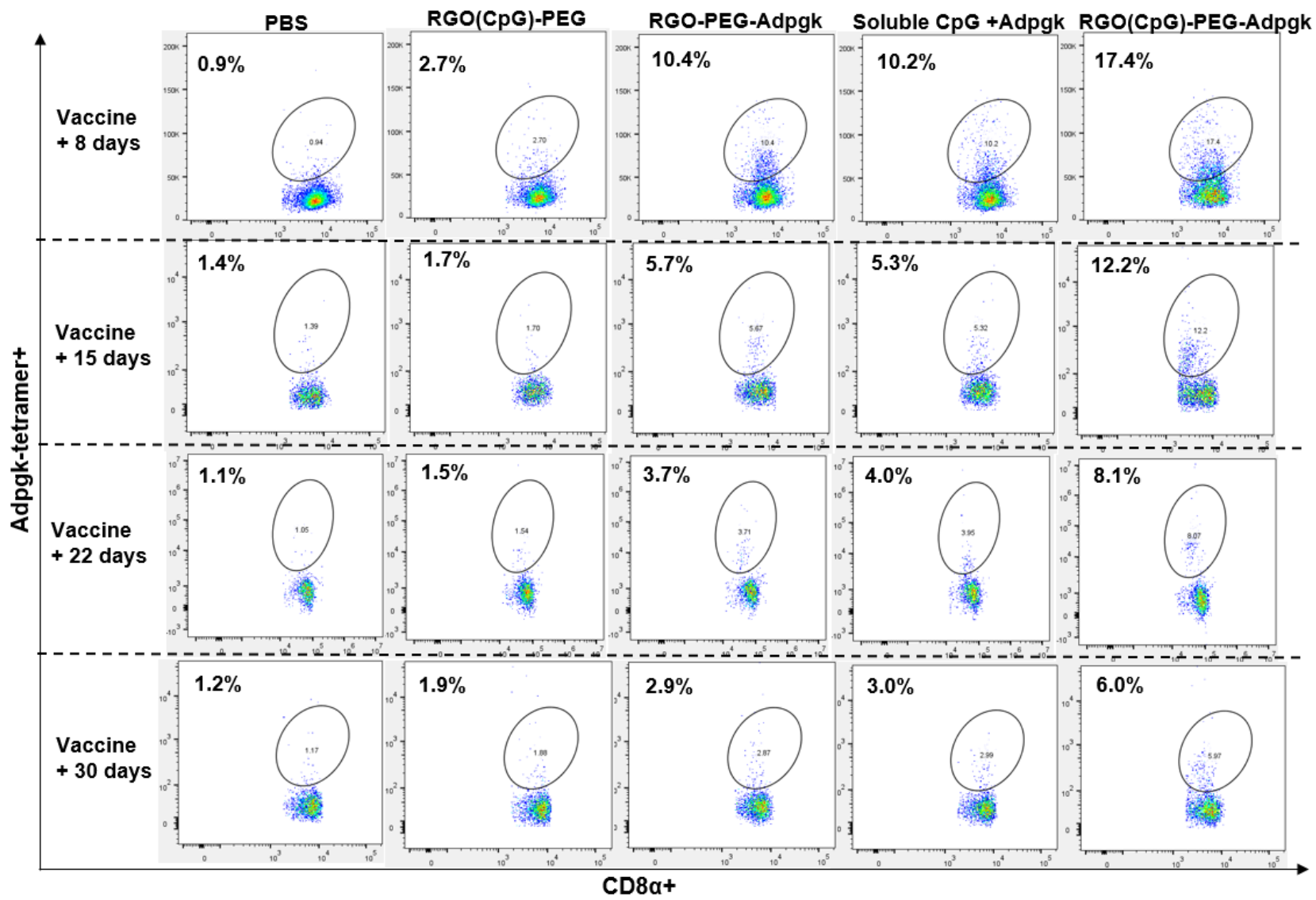

Figure S7. The representative scatter plots and the frequencies of Adpgk-specific CD8 $\alpha^{+} T$-cells in peripheral blood in animals treated as in Figure $\mathbf{4 a .}$ 


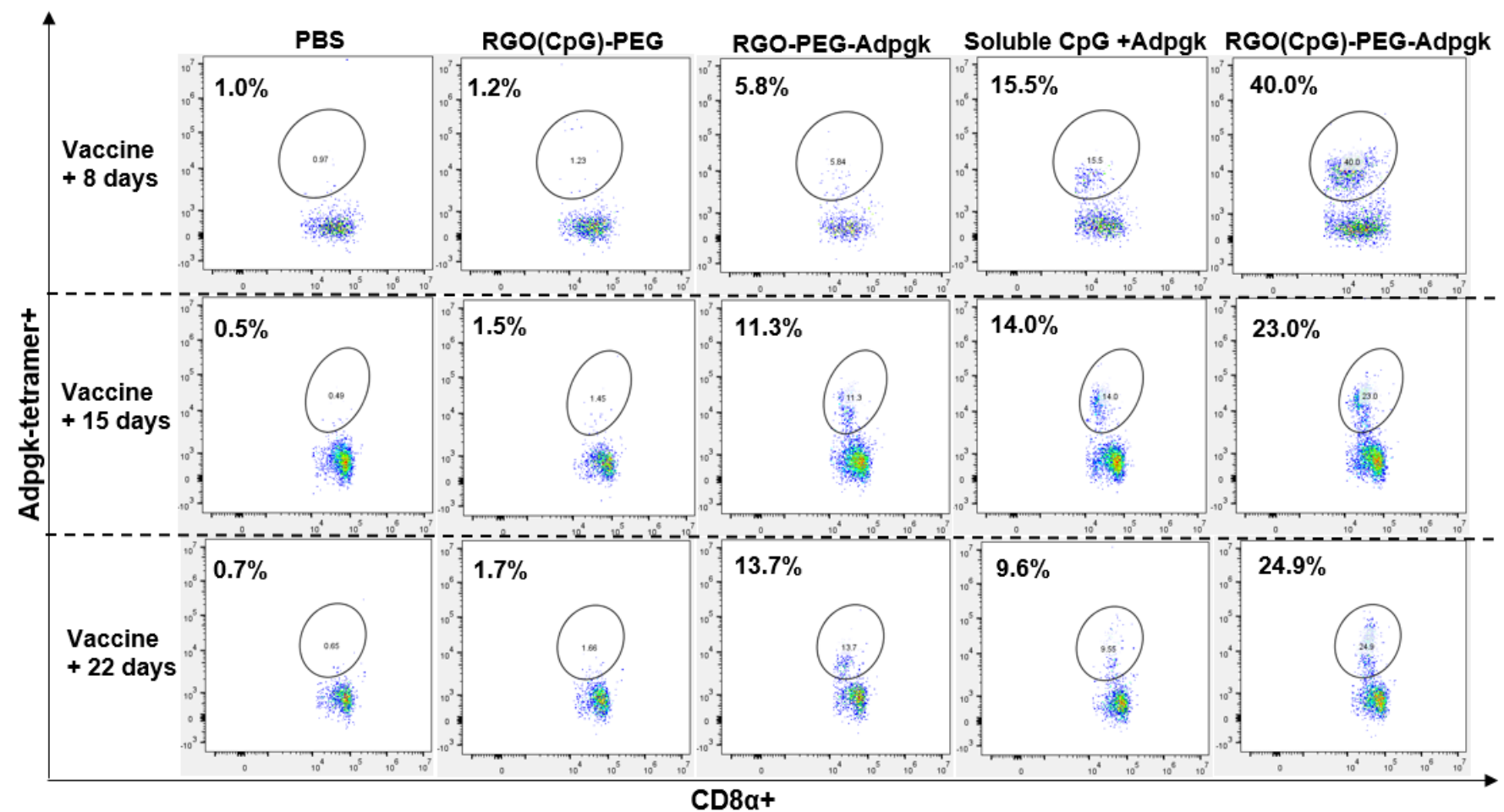

Figure S8. The representative scatter plots and frequencies of Adpgk-specific CD8 $\alpha^{+}$T-cells in peripheral blood in animals treated as in Figure 4e.

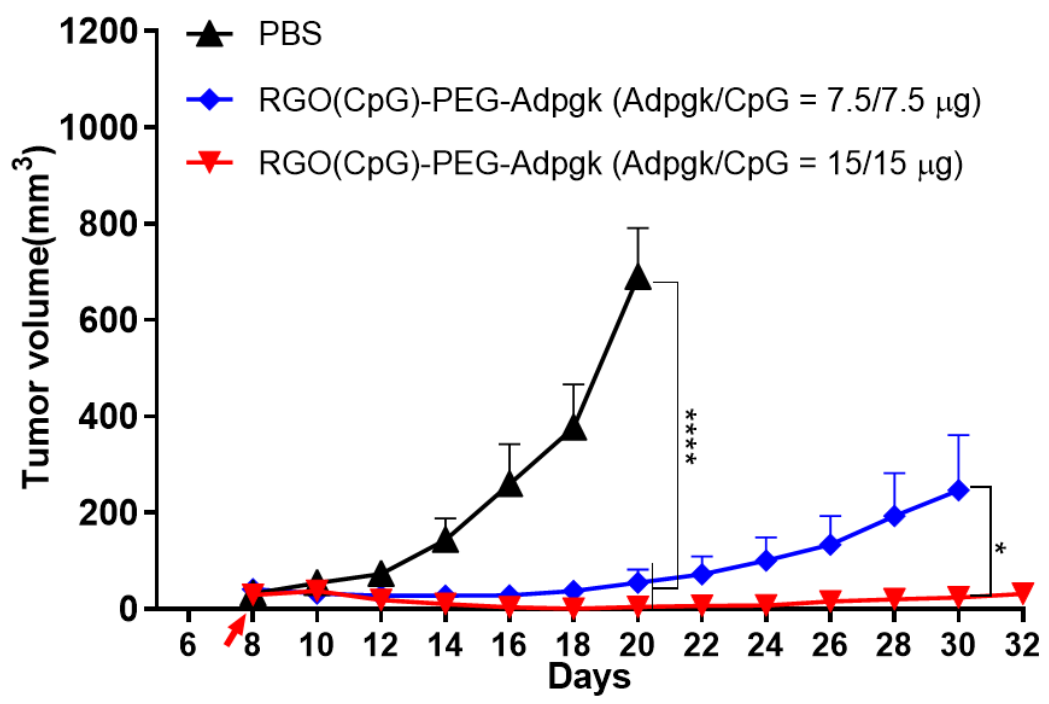

Figure S9: Dose-dependent efficacy of RGO(CpG)-PEG-Adpgk vaccine in MC-38 colon carcinoma-bearing mice. Average tumor growth curve of (1) PBS control; (2) RGO(CpG)-PEG-Adpgk treatment group (Adpgk and CpG doses = $7.5 \mu \mathrm{g}$ each); (3) RGO(CpG)-PEG-Adpgk treatment group (Adpgk and CpG doses = $15 \mu \mathrm{g}$ each). Mice were vaccinated by SC at tail base for once at Day 8. Data represent mean \pm SEM from a representative experiment $(n=5)$. Data was analyzed by two-way ANOVA with Tukey's HSD multiple comparison post hoc test ${ }^{*} \mathrm{P}<0.05,{ }^{* * *} \mathrm{P}<0.0001$. 
(a)

\begin{tabular}{|c|c|c|c|c|c|c|}
\hline Name & Reference range & PBS & Soluble CpG + Adpgk & RGO(CpG)-PEG & RGO-PEG-Adpgk & RGO(CpG)-PEG-Adpgk \\
\hline AST, U/L & $39.6-386.1$ & $33.8 \pm 10.3$ & $71.0 \pm 24.9$ & $43.4 \pm 10.8$ & $41.6 \pm 7.6$ & $45.6 \pm 4.2$ \\
\hline $\mathrm{ALT}, \mathrm{U} / \mathrm{L}$ & $24.3-115.3$ & $38.0 \pm 5.1$ & $47.5 \pm 10.6$ & $37.6 \pm 9.9$ & $39.5 \pm 8.4$ & $51.5 \pm 5.7$ \\
\hline ALP, U/L & $65.5-364.2$ & $131 \pm 16.9$ & $187 \pm 17.7$ & $140 \pm 14.3$ & $138 \pm 12.8$ & $176 \pm 14.2$ \\
\hline Triglyceride, mg/dL & $72.7-303.2$ & $79.5 \pm 22.1$ & $82.1 \pm 18.6$ & $75.1 \pm 7.9$ & $82.8 \pm 16.7$ & $70.6 \pm 14.9$ \\
\hline Cholesterol, mg/dL & $60.2-167.3$ & $72.0 \pm 14.8$ & $61.0 \pm 13.7$ & $62.4 \pm 10.7$ & $65.8 \pm 9.7$ & $61.2 \pm 10.3$ \\
\hline Glucose, mg/dL & 79.4-354.7 & $248 \pm 34.1$ & $245 \pm 27.1$ & $198 \pm 34.2$ & $183 \pm 15.9$ & $156.8 \pm 17.2$ \\
\hline Creatine phosphokinase, U/L & $22.0-198.0$ & $95.2 \pm 24.9$ & $114 \pm 30.3$ & $93.6 \pm 12.3$ & $90.0 \pm 10.6$ & $113 \pm 12.3$ \\
\hline Blood urea nitrogen, $\mathrm{mg} / \mathrm{dL}$ & $5.2-30.7$ & $29.5 \pm 8.6$ & $33.5 \pm 5.4$ & $22.5 \pm 3.6$ & $30.1 \pm 8.1$ & $26.6 \pm 8.9$ \\
\hline
\end{tabular}

(b)
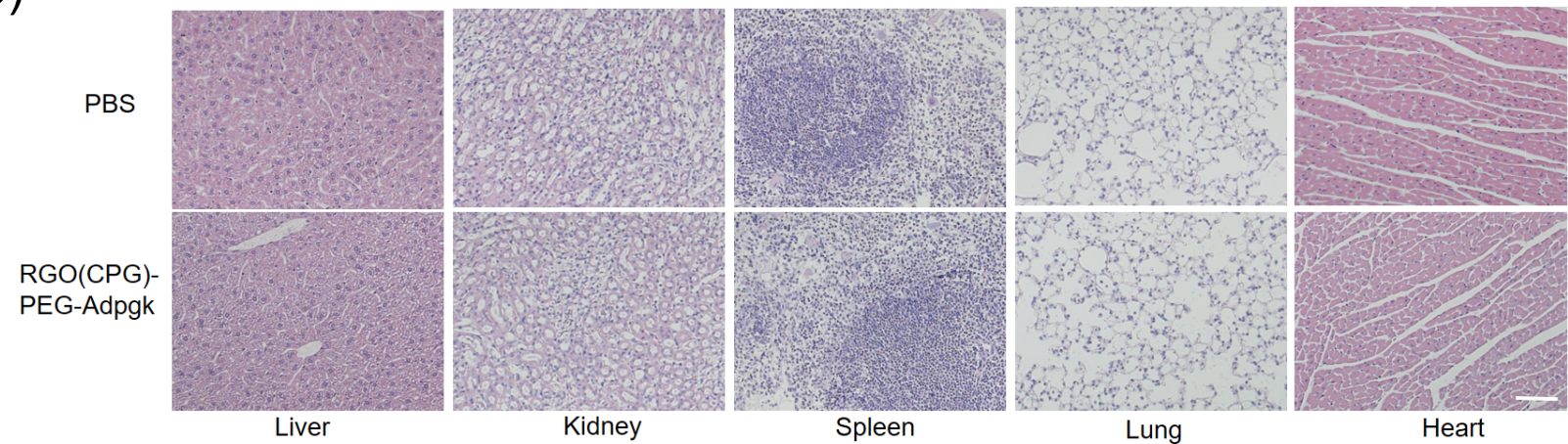

Figure S10. a, Analyses of serum biochemical markers after 7 days of vaccination with the indicated groups. b, Hematoxylin-eosin (H\&E) staining images of major organs after 15 days of SC vaccination with PBS or RGO(CpG)-PEG-Adpgk.
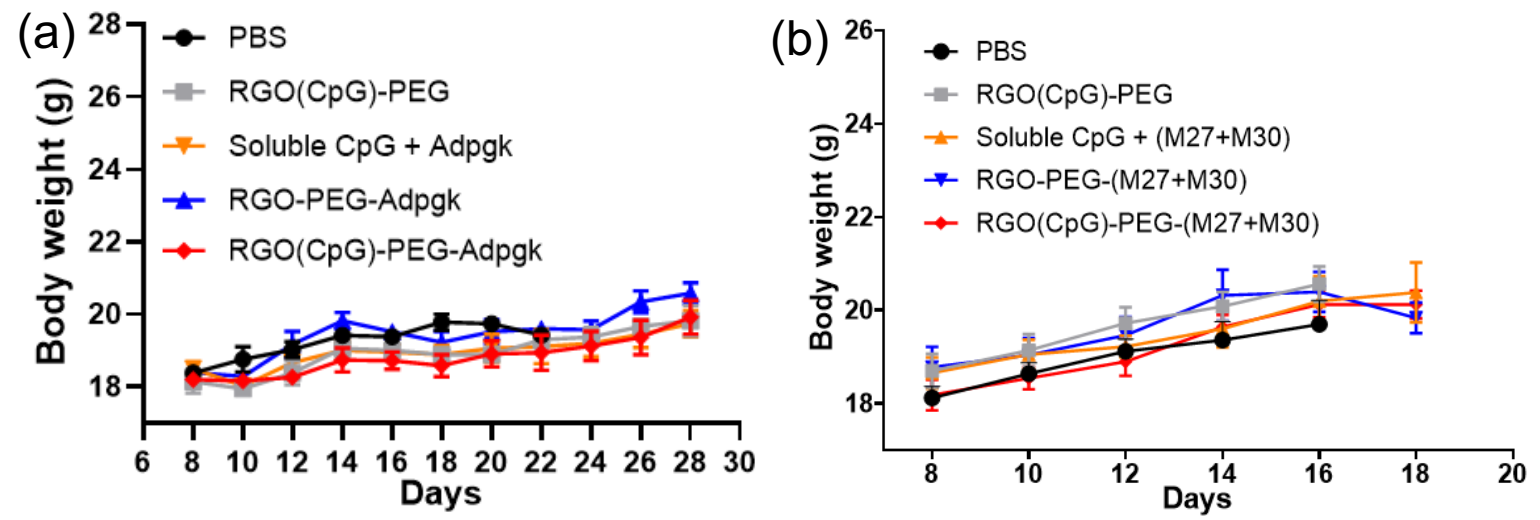

Figure S11. a, Body weight of MC-38 tumor-bearing mice treated as in Figure 4e. b, Body weight of B16F10 tumor-bearing mice treated as in Figure 5a. 